\title{
The Flash Oxidation (FOX) System: A Novel Laser-Free Fast Photochemical Oxidation Protein Footprinting Platform
}

\section{Supporting Information}

Joshua S. Sharp ${ }^{1,2,3}$, Emily E. Chea ${ }^{1}$, Sandeep K. Misra ${ }^{2}$, Ron Orlando ${ }^{1,4,5}$, Marla Popov ${ }^{5}$, Robert W. Egan $^{1}$, David Holman ${ }^{1}$ and Scot R. Weinberger ${ }^{1}$

1. GenNext Technologies, Inc., Half Moon Bay, CA 94019

2. Department of BioMolecular Sciences, School of Pharmacy, University of Mississippi, University, Mississippi 38677

3. Department of Chemistry and Biochemistry, University of Mississippi, University, Mississippi 38677

4. Complex Carbohydrate Research Center, University of Georgia, Athens, GA 30602

5. GlycoScientific, Athens, GA 30602

*Correspondence to Joshua S. Sharp, jsharp@olemiss.edu 


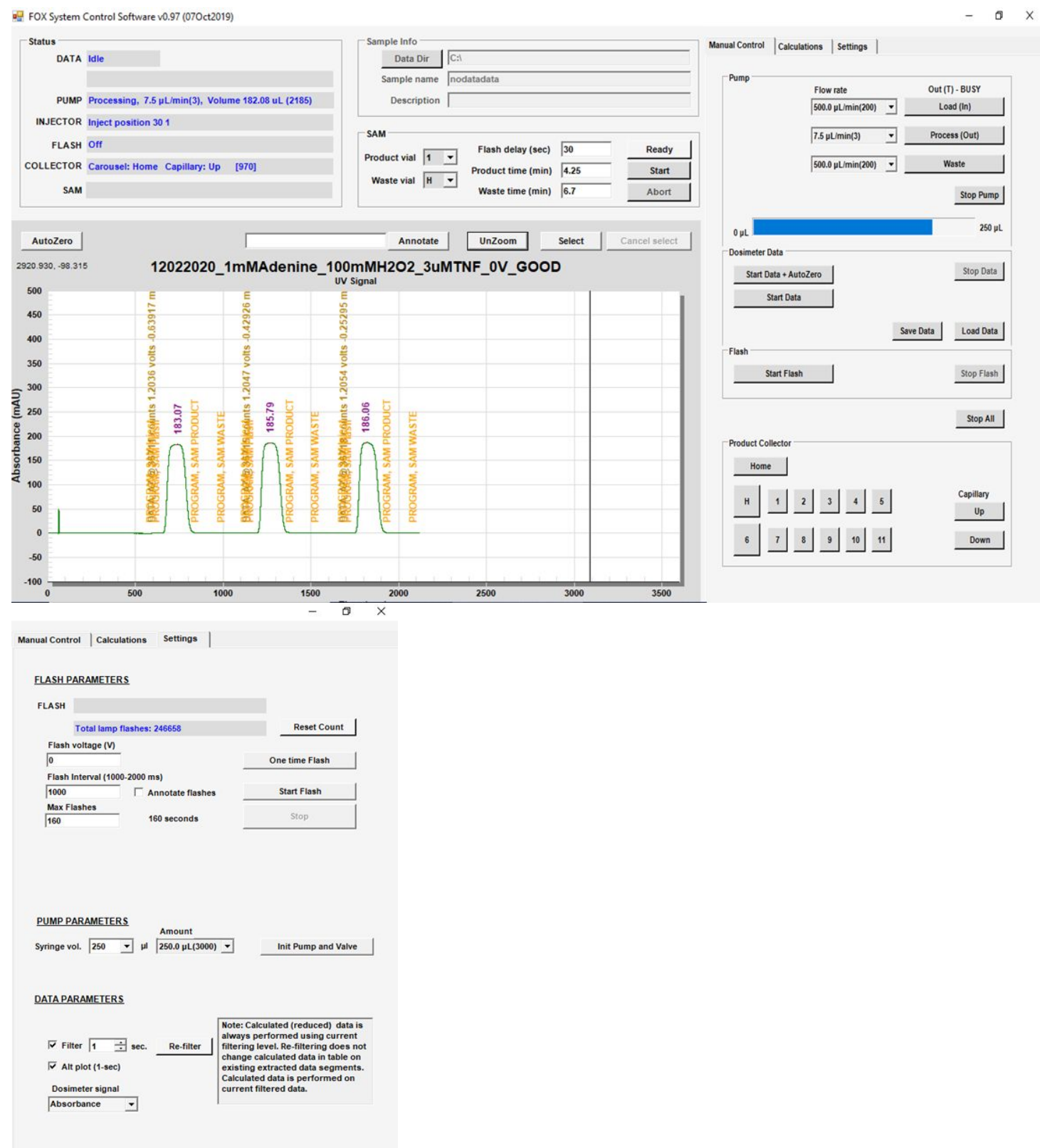

Figure S1. FOX photolysis system control software sample screens. Top: Main real time monitoring and control screen. Inline adenine radical dosimetry readouts are plotted in real time, and all modules and sample collector can be controlled manually or via a pre-determined timing program. Bottom:

Subscreen for setting parameters for the flow injection pump, the FOX flash cell module power supply, and the dosimetry module data acquisition. 


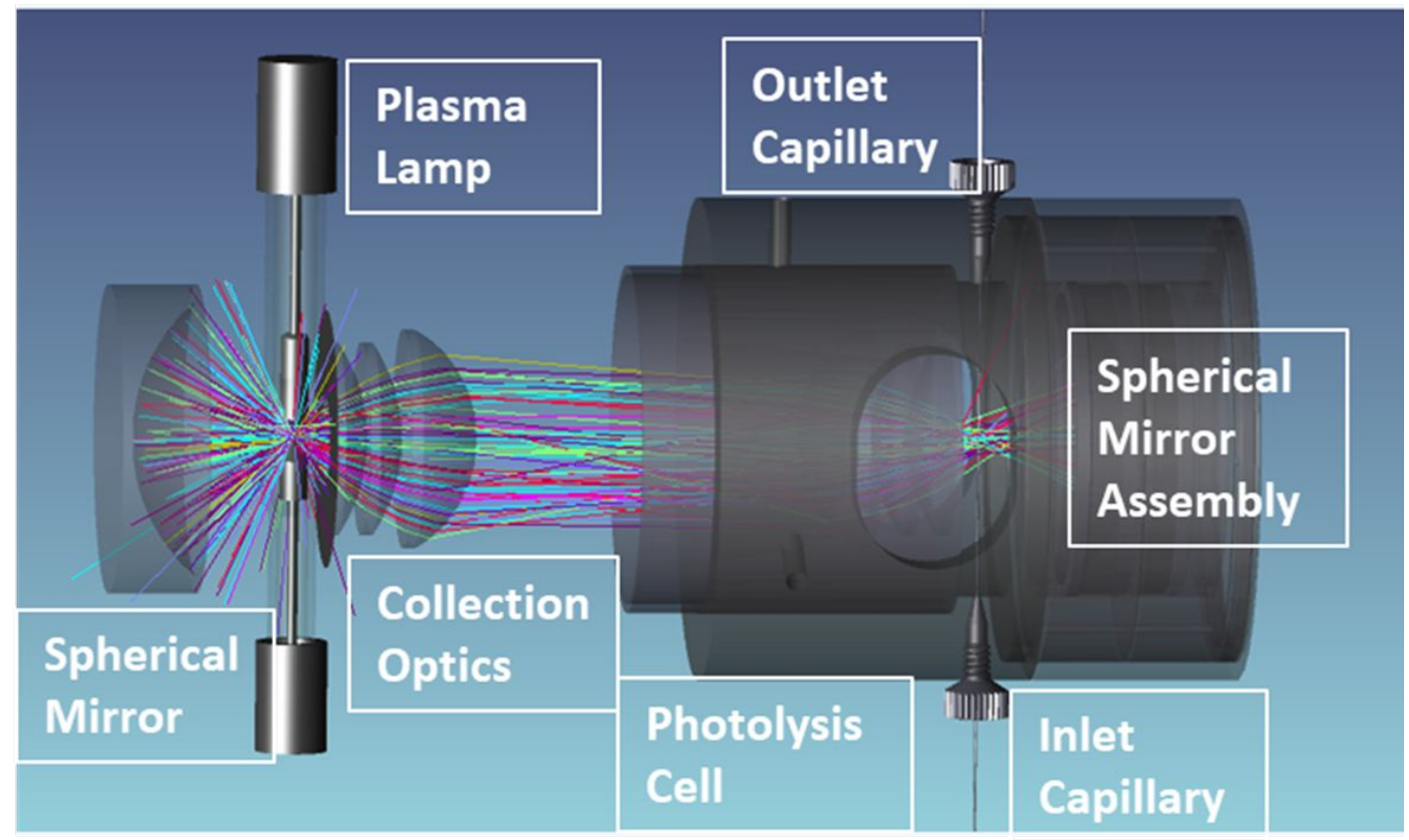

Figure S2. Conceptual diagram of the integrated optics for the FOX photolysis cell. Radiant light from the high-pressure flash lamp is collected via a spherical mirror on the distal side of the lamp, and a set of collection optics on the proximal side. Collected light is focused into the lumen of the fused silica capillary traversing the optical cell vertically, parallel to the orientation of the plasma lamp electrodes to maximize the volume covered by each flash. Light that passes through the capillary is returned via a spherical mirror assembly to the rear of the capillary, almost doubling the illumination of the sample. 

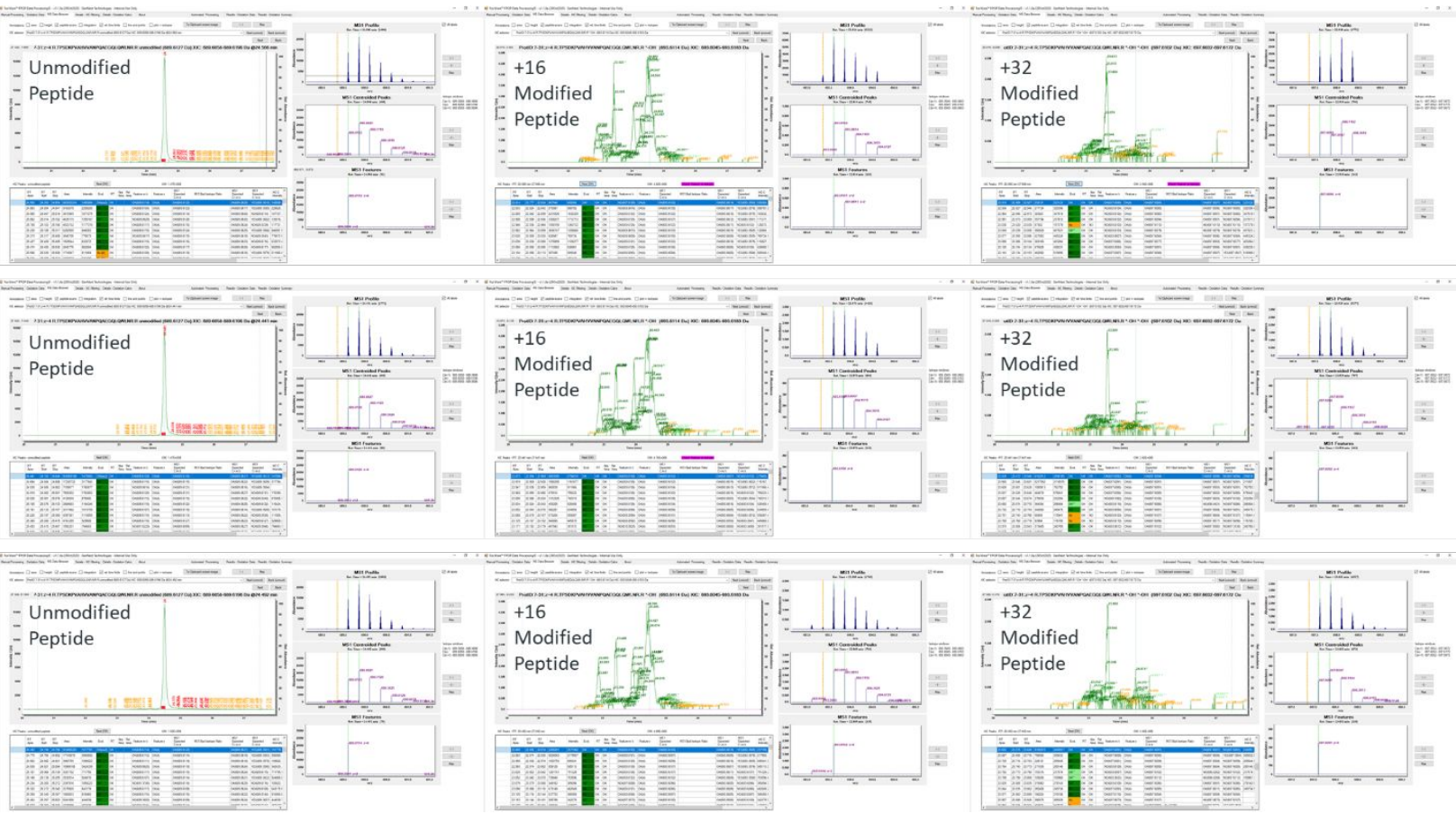

Figure S3. FoxWare Protein Footprinting Software output for three replicates of peptide 7-31. Left: Unmodified peptide as identified by Byonic. Middle: $\mathrm{M}+\mathrm{O}$ product as identified by the FoxWare Protein Footprinting software. Right: $\mathrm{M}+2 \mathrm{O}$ products as identified by the FoxWare Protein Footprinting software. Green annotations above a peak indicate the peak was accepted as an oxidation product, while yellow annotations indicate the peak failed at least one of the quality check criteria. Peak reproducibility across replicates is very good for both sets of oxidation products. 

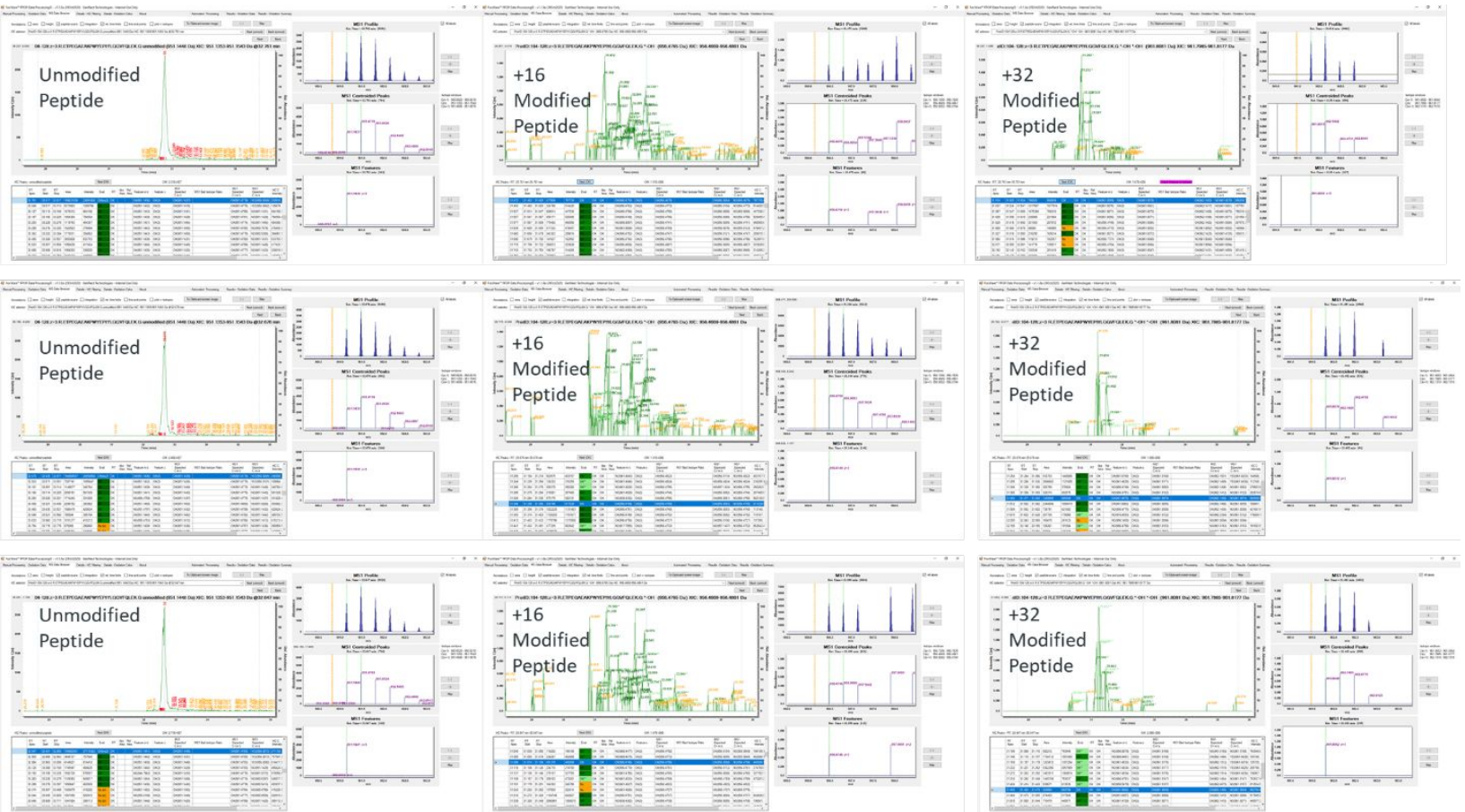

Figure S4. FoxWare Protein Footprinting Software output for three replicates of peptide 104-128. Left: Unmodified peptide as identified by Byonic. Middle: $\mathrm{M}+\mathrm{O}$ product as identified by the FoxWare Protein Footprinting software. Right: $\mathrm{M}+2 \mathrm{O}$ products as identified by the FoxWare Protein Footprinting software. Green annotations above a peak indicate the peak was accepted as an oxidation product, while yellow annotations indicate the peak failed at least one of the quality check criteria. Peak reproducibility across replicates is very good for both sets of oxidation products. 

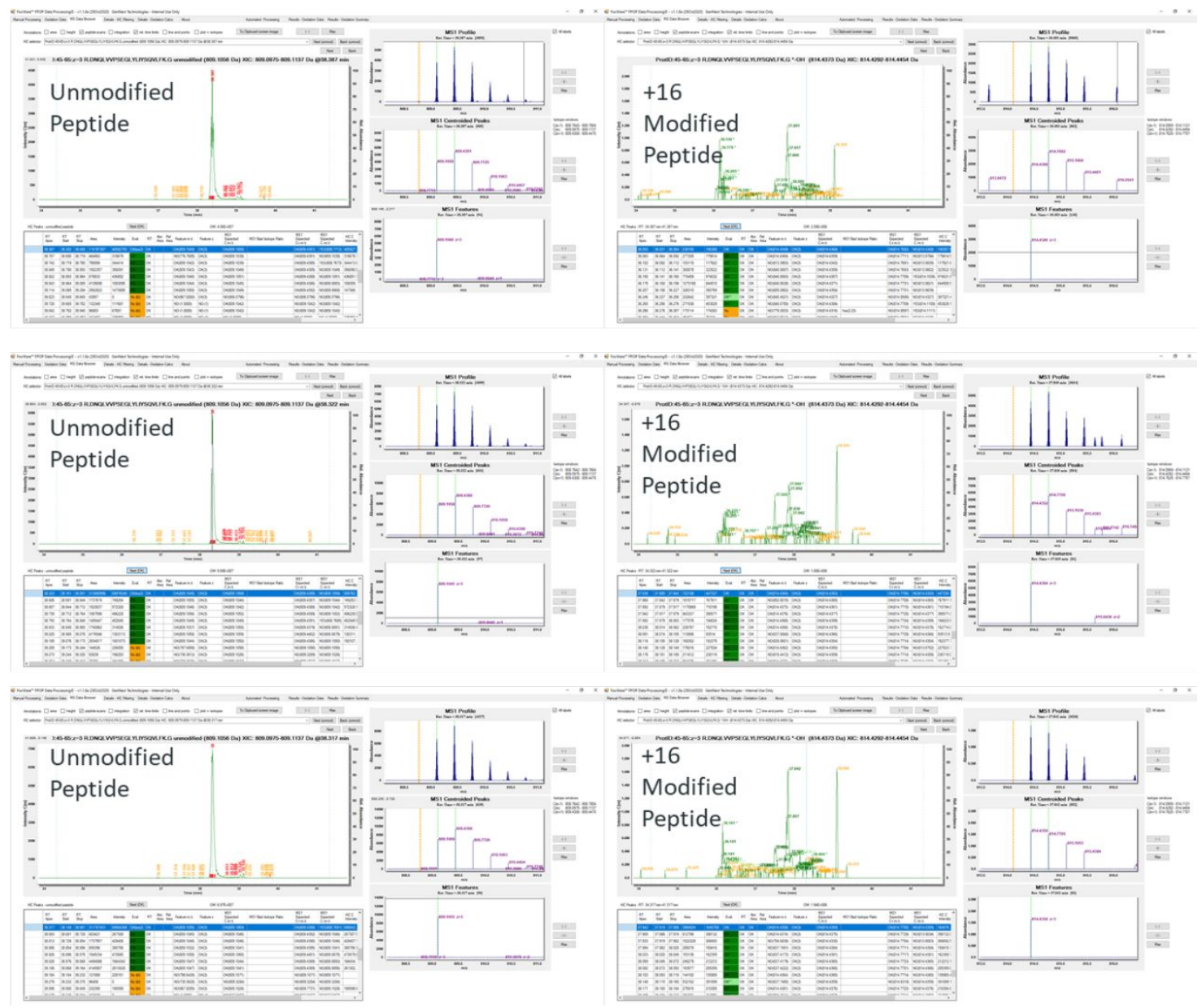

Figure S5. FoxWare Protein Footprinting Software output for three replicates of peptide 45-65. Left: Unmodified peptide as identified by Byonic. Right: $\mathrm{M}+\mathrm{O}$ product as identified by the FoxWare Protein Footprinting software. Green annotations above a peak indicate the peak was accepted as an oxidation product, while yellow annotations indicate the peak failed at least one of the quality check criteria. Peak reproducibility across replicates is very good for the $\mathrm{M}+\mathrm{O}$ oxidation products. 


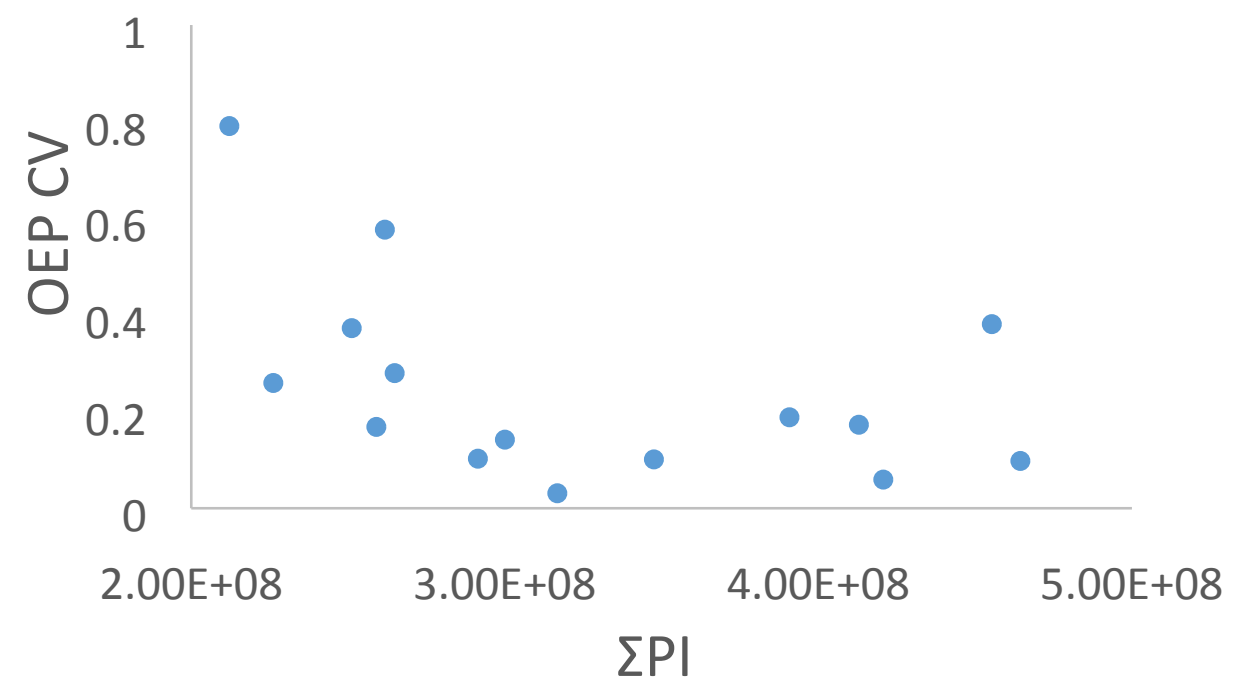

Figure S6. FOX Oxidation Events per Peptide Coefficient of Variation vs the sum of peptide intensity for both unoxidized and all detected oxidized variations of the peptide. 